Revue internationale P.M.E.

Économie et gestion de la petite et moyenne entreprise

\title{
Les caractéristiques et les spécificités de la PME : favorables ou défavorables au juste-à-temps?
}

\section{René Gélinas, Alain Halley, Real Jacob et Jocelyn Drolet}

Volume 9, numéro 2, 1996

URI : https://id.erudit.org/iderudit/1008263ar

DOI : https://doi.org/10.7202/1008263ar

Aller au sommaire du numéro

Éditeur(s)

Presses de l’Université du Québec

ISSN

0776-5436 (imprimé)

1918-9699 (numérique)

Découvrir la revue

Citer cette note

Gélinas, R., Halley, A., Real Jacob \& Drolet, J. (1996). Les caractéristiques et les spécificités de la PME : favorables ou défavorables au juste-à-temps? Revue internationale P.M.E., 9(2), 81-101. https://doi.org/10.7202/1008263ar
Résumé de l'article

L'implantation du juste-à-temps (JAT) dans les PME représente un défi important lié au management moderne. De plus en plus de PME subissent les pressions des grandes entreprises manufacturières pour modifier leur mode traditionnel de gestion, tant sur le plan opérationnel qu'organisationnel, en faveur du système favorisant les transactions en flux tendus qu'est le JAT. Dans la littérature, on discute abondamment de sujets tels que les facteurs de succès du JAT ou les conditions d'une implantation réussie ; mais il s'avère que la majorité des études abordent le JAT sous l'angle de la grande entreprise. Considérant l'importance de la PME dans l'économie des pays industrialisés et constatant qu'un nombre sans cesse croissant de ces PME verront leur mode de gestion modifié au profit du JAT, il nous apparaît pertinent d'analyser les caractéristiques de la PME afin de cibler celles qui sont favorables ou défavorables au JAT. 


\title{
Les caractéristiques et les spécificités de la PME: favorables ou défavorables au juste-à-temps?
}

\author{
René GÉLINAS \\ Alain HALLEY \\ Réal JACOB \\ Jocelyn DROLET \\ Université du Québec à Trois-Rivières
}

MOTS CLÉS

JAT - PME - Implantation - Facteurs de succès - Spécificité

\begin{abstract}
RÉSUMÉ
L'implantation du juste-à-temps (JAT) dans les PME représente un défi important lié au management moderne. De plus en plus de PME subissent les pressions des grandes entreprises manufacturières pour modifier leur mode traditionnel de gestion, tant sur le plan opérationnel qu'organisationnel, en faveur du système favorisant les transactions en flux tendus qu'est le JAT. Dans la littérature, on discute abondamment de sujets tels que les facteurs de succès du JAT ou les conditions d'une implantation réussie; mais il s'avère que la majorité des études abordent le JAT sous l'angle de la grande entreprise. Considérant l'importance de la PME dans l'économie des pays industrialisés
\end{abstract}

\section{LES AUTEURS}

René Gélinas détient un Ph.D. de l'Université Laval en opérations et systèmes de décisions. II est professeur à l'Université du Québec à Trois-Rivières. Ses travaux de recherche portent sur le juste-à-temps dans les PME et sur les méthodes statistiques de contrôle des procédés. II a écrit dans plusieurs revues dont European Journal of Purchasing and Supply Management, International Journal of Quality and Reliability Management et Journal of the Operation Research Society.

Alain Halley, M.Sc. en gestion des PME de I'Université du Québec à Trois-Rivières. II est étudiant au doctorat en logistique et organisation à l'Université de la Méditerranée (Aix-Marseille II). Boursier de la Chaire Bombardier Sea-Doo/Ski-Doo en gestion du changement technologique dans les PME, il est professionnel de recherche au GREPME depuis 1993. II s'intéresse à la logistique d'approvisionnement et de distribution et à l'intégration logistique. 
et constatant qu'un nombre sans cesse croissant de ces PME verront leur mode de gestion modifié au profit du JAT, il nous apparaît pertinent d'analyser les caractéristiques de la PME afin de cibler celles qui sont favorables ou défavorables au JAT.

\begin{abstract}
Just-in-Time (JIT) implementation in small businesses (SMB) is becoming an important challenge with respect to modern management. More and more SMBs must cope with large businesses pressuring them to modify their operational and organizational structure by adjusting it to JIT prerequisites. The literature is abundant concerning issues like JIT success factors or guidelines for a successful implementation but most of the studies consider large businesses in their JIT discussions. Given the paramount importance of SMBs in industrialized countries and considering that an increasing number of SMBs will be affected by JIT, it seems appropriate to analyze the characteristics and specificities of the SMB in order to target those favorable or unfavorable to JIT.
\end{abstract}

\title{
RESUMEN
}

La implantación de Justo-a-Tiempo en las pequeñas y medianas empresas (PyMEs) representa un reto importante ligado a la gestión moderna. Cada vez más las PyMEs sufren presiones de grandes empresas manufactureras para modificar su modo de gestión tradicional, tanto en el plan operacional como organisacional, en favor de un sistema que favorezca las transacciones en flujo sincronizado. La literatura discute abundantemente los temas tales como los factores de éxito de Justo-a-Tiempo o las condiciones de una implantación exitosa del mismo, pero la mayoría de estos estudios abordan el Justo-aTiempo con el ángulo de la gran empresa. Considerando la gran importancia de la PyMEs en las economías de los paises industrializados y constatando

Réal Jacob, M.Ps, C.E.A. en sciences du travail, est directeur du GREPME (Groupe de recherche en économie et gestion des $P M E$ ), chercheur principal de la Chaire Bombardier Sea-Doo/Ski-Doo en gestion du changement technologique dans les PME et professeur au Département des sciences de la gestion et de l'économie de l'Université du Québec à Trois-Rivières. II travaille sur les nouveaux modes de gestion, la flexibilité organisationnelle, l'entreprise réseau et la gestion du changement. II a codirigé récemment l'ouvrage intitulé Changement technologique et gestion des ressources humaines: fondements et pratiques, 1995, Gaëtan Morin Éditeur.

Jocelyn Drolet, ing., Ph.D. en génie industriel de l'Université Purdue, États-Unis. II est professeur en génie industriel à l'Université du Québec à Trois-Rivières depuis 1989 et membre de la Chaire Bombardier Sea-Doo/Ski-Doo en gestion du changement technologique dans les PME. II s'intéresse à la gestion de production assistée par ordinateur, au juste-à-temps et d'une manière plus générale aux techniques modernes de planification et de gestion des opérations. II a donné plusieurs conférences au Canada, aux États-Unis et en Europe et a publié plus de 40 articles scientifiques. Il est membre de l'Ordre des ingénieurs du Québec et de l'Association internationale de génie industriel.

Adresse : Chaire Bombardier Sea-Doo/Ski-Doo en gestion du changement technologique dans les PME, Université du Québec à Trois-Rivières, C.P. 500, Trois-Rivières, Québec, G9A $5 \mathrm{H} 7$. 
que el número creciente de estas PME que modificarán su modo de gestión en beneficio del Justo-a-Tiempo, consideramos pertinente el analizar las características y especificaciones de la PME con el fin de detectar aquellas que sean favorables o bien desfavorables a el Justo-a-Tiempo.

\section{Les PME et l'implantation du JAT' : les enjeux}

Les entreprises à succès d'aujourd'hui savent à quel point il est primordial de s'adapter aux besoins du consommateur pour conserver leur part de marché et assurer leur croissance. De la même façon, les grandes orientations stratégiques des PME manufacturières sont indéniablement influencées par les nouvelles exigences des grandes entreprises manufacturières (GEM). Il n'est donc guère surprenant de constater que certaines GEM opérant en JAT exercent de fortes pressions sur leurs fournisseurs, souvent des PME, pour qu'ils adoptent à leur tour ce mode de production (Landry et Trudel, 1993 ; Bérard, 1992). Les GEM doivent en effet compter sur un réseau de fournisseurs dits de «classe mondiale » pour supporter leur procédé de fabrication dans l'implantation du JAT (Landry, 1990). Cependant, développer et supporter des fournisseurs de classe mondiale signifie plus que demander des livraisons en petits lots ou participer à quelques séances de formation. Landry (1990) considère que la gestion efficace des sources d'approvisionnement en contexte de JAT doit s'appuyer sur le développement à long terme des fournisseurs afin de mieux exploiter leurs expertises et de rehausser leurs capacités de design, de fabrication et de distribution au niveau de processus de classe mondiale. La réduction du nombre de fournisseurs et la réalisation d'ententes à long terme sont évidemment deux facteurs essentiels à la réussite de cette gestion. Les PME retenues bénéficieront d'ailleurs de plusieurs avantages (engagement à long terme, augmentation de la part de marché, transfert de connaissances, etc.), mais également de certains inconvénients (risque de quasi-intégration, dépendance unilatérale, conflit occasionné par la répartition des bénéfices tirés de la coopération, etc.). À la lumière de ces informations, la question qui se pose est de savoir quelles raisons amènent les PME à rechercher une transposition des méthodes utilisées par les grandes entreprises, notamment le JAT (ou flux tendus)?

Une partie de la réponse à cette question se trouve à l'origine même de la décision d'implanter le JAT dans les PME, c'est-à-dire pour répondre aux attentes de la GEM opérant en JAT. Pour ce faire, les PME désirent adopter un processus de production complémentaire à celui de la firme acheteuse afin

1. Tout au long de cet article, le générique JAT fera référence au mode de gestion intégré à la totalité de la chaîne logistique (approvisionnement, production, livraison, etc.). 
de synchroniser les flux et maximiser l'efficience du processus global. Dans ce contexte, le fournisseur est considéré comme un prolongement de la firme cliente et les fournisseurs doivent développer l'expertise nécessaire afin d'offrir un produit compétitif et à moindre coût. Pour permettre aux fournisseurs d'accéder à un tel niveau de compétences, les donneurs d'ordres (GEM) doivent valoriser l'intégration de ces ressources critiques au processus global de fabrication (Landry, 1990). Les fournisseurs seront de cette manière appelés à intervenir lors des étapes de conception du produit afin d'échanger les connaissances techniques et technologiques permettant la réduction des coûts de fabrication. La coopération des deux parties est importante pour l'atteinte d'un des principaux objectifs du JAT : l'élimination du gaspillage.

Le fait que peu d'articles traitent essentiellement de l'implantation du JAT chez les PME explique également que celles-ci se réfèrent au modèle d'implantation utilisé par les GEM. Il ne faut pas oublier que les GEM sont les instigatrices de cette modification des procédés de production. De plus, en utilisant un modèle d'implantation similaire à celui utilisé par la firme acheteuse, la PME est en mesure de lui demander un soutien organisationnel. Les deux parties impliquées dans la métamorphose des processus de fabrication ont effectivement beaucoup à gagner à coopérer. Potter (1990) confirme l'importance que revêt cette double implication en indiquant qu'un partenariat réussi requiert de la planification, une implantation organisée, de la diplomatie et beaucoup de travail. De plus, il faut se rappeler que la réduction du nombre de fournisseurs amène le donneur d'ordres opérant en JAT à choisir ses fournisseurs privilégiés (fournisseurs ou sous-traitants de premier ordre). Dans la mesure où l'évaluation des fournisseurs est devenue une notion importante dans la gestion des approvisionnements en JAT (Offodile et Arrington, 1992), les critères utilisés lors de la sélection correspondent inévitablement aux attentes de la firme acheteuse, d'où l'importance, pour la PME intéressée à conserver sa part de marché, d'implanter un mode de production compatible à celui de la GEM.

La sommation des différentes activités d'intégration et de coopération vont conséquemment modifier les relations observables entre les PME et les GEM opérant en JAT. De ce pas, on assistera au passage de la sous-traitance de capacité à la sous-traitance «d'intelligence ». Dans cette optique, les soustraitants (fournisseurs) sont considérés à la fois comme des spécialistes de la production (capacité), mais également comme des spécialistes de la conception et de l'innovation. Cette nouvelle perception des relations interorganisationnelles permet aux donneurs d'ordres d'exprimer des besoins génériques et non plus uniquement des requêtes quantitatives (Chaire Bombardier Sea-Doo/ Ski-Doo, 1995). Les sous-traitants de premier ordre auront ainsi pour mission de gérer les sous-traitants de deuxième et troisième ordre afin de produire et 
livrer à temps les multiples composantes nécessaires à la fabrication (ou à l'assemblage) des produits par le donneur d'ordres. À travers cette restructuration des relations entre les différents sous-traitants et le donneur d'ordres se dessine l'entreprise en réseau. Ce réseau d'entreprises favorise le passage de la relation traditionnelle de subordination vers une relation d'équivalence relative, où chaque firme tendra à faire valoir ses propres compétences distinctives et complémentaires.

Un autre élément qui alimente la complexité de l'environnement externe de la PME est l'abolition des barrières économiques. L'entreprise manufacturière contemporaine est constamment agressée par des pressions associées tant à la demande qu'à l'offre. De fait, l'élargissement des marchés (la demande) exige à la fois un accroissement des capacités productives et une diminution du temps de réponse. À l'opposé, l'offre subit également des modifications majeures. Désormais, il faut prendre en considération l'environnement concurrentiel qui s'avère de plus en plus féroce et qui exige des efforts importants au regard de la réduction des coûts et du développement ou de l'accroissement des compétences distinctives. L'entreprise de classe mondiale a évidemment agrandi son champ d'action territoriale, mais une telle situation devra être analysée sous l'angle de l'ouverture concurrentielle mondiale. Le défi est de taille et fait appel au dépassement et à l'innovation dans son expression la plus pure afin d'élargir les horizons de l'organisation. La PME opérant en JAT dans un contexte d'entreprises en réseau bénéficiera, à certaines conditions, d'une panoplie d'avantages déterminants lui permettant d'accéder au statut d'innovateur, voire de leader sectoriel. À ce chapitre, la réduction des coûts de fabrication, l'augmentation de la flexibilité et l'élimination de toute forme de gaspillage sont quelques exemples d'éléments qui permettent le développement de compétences distinctives et déterminantes pour l'entreprise.

\section{L'importance de l'analyse des caractéristiques et des spécificités des PME par rapport au JAT}

L'importance de l'analyse des caractéristiques et des spécificités des PME par rapport au JAT ne se limite pas au seul plan théorique. D'une part, et tel que nous l'avons déjà mentionné, un nombre limité de publications traite de façon spécifique du JAT en contexte PME et, d'autre part, les statistiques indiquent que les PME constituent un agent économique majeur pour les pays industrialisés (voir Acs, 1992 ; Light, 1993). De plus, les grandes entreprises manufacturières (GEM) telles Bombardier, Toyota et GM sont à la tête d'un impressionnant réseau de fournisseurs qui sont, pour la plupart, des PME. À titre d'exemple, la seule division Sea-Doo / Ski-Doo de Bombardier à Valcourt au Québec est le centre de gravité d'un réseau de plus de 250 fournisseurs qui 
ont eux-mêmes leur propre réseau de fournisseurs de deuxième rang. Désormais d'une importance reconnue par un nombre grandissant de chercheurs et considérée comme une institution spécifique à l'opposé des systèmes de grande dimension (Julien et Marchesnay, 1988 ; GREPME, 1994), la PME est donc une constituante majeure des réseaux de fournisseurs des grandes entreprises manufacturières (Gélinas et al., 1994a). Or, il existe souvent un rapport de force entre les GEM et les PME qui tend à conditionner les comportements des PME en fonction des attentes des GEM par le biais des pratiques de gestion de celles-ci (Landry et Trudel, 1993). Les GEM exercent ainsi une pression sur les PME obligeant ces dernières à acquérir un avantage concurrentiel par différenciation sans qu'elles puissent compenser par des coûts supplémentaires (Porter, 1991).

Donc, si d'une part les GEM s'intéressent de plus en plus au JAT afin d'accroître leur compétitivité (Mehra et Inman, 1992 ; Delmas et Roy, 1988²) et que, d'autre part, cet intérêt a un impact sur les $\mathrm{PME}^{3}$, il est clair que le nombre de PME qui auront à adopter le JAT augmentera. Considérant le rôle de premier plan joué par les PME dans les pays industrialisés ${ }^{4}$, l'analyse des enjeux du JAT pour ces entreprises revêt une grande importance tout en représentant un défi de taille du fait de leurs spécificités. Il convient donc, pour favoriser une meilleure compréhension de ces enjeux, de faire ressortir ces spécificités à travers certains grands axes de différenciation PME/GEM, de mettre en évidence les effets et les implications de ces spécificités sur le JAT et, finalement, de cibler celles qui pourraient éventuellement s'avérer favorables et défavorables au JAT.

L'étude des caractéristiques et spécificités des PME au regard du JAT est basée sur une analyse de la documentation traitant, aussi bien de façon empirique que théorique, des différences entre PME et GEM, ainsi que du JAT en contexte de PME. De fait, en plus de mettre en relief le JAT par rapport aux caractéristiques propres des PME, cet article contribue, par la revue de la

2. Selon cette étude, trois ensembles de facteurs contribuent à amoindrir l'efficacité de l'industrie américaine : la gestion des ressources humaines, la gestion du temps et surtout l'organisation $d u$ processus productif.

3. Brown et Mitchell (1991) ont répertorié les GEM ayant mis en place le JAT : Black \& Decker Manufacturing Company, Exxon Corporation, General Electric Company, Hewlett-Packard Company et Xerox Corporation. Sachant qu'à elles seules, ces six GEM transigent avec au moins une dizaine de milliers d'autres entreprises (souvent des PME), il devient difficile de nier l'effet « contagieux » des modes de gestion des GEM.

4. Elles regroupent $72,1 \%$ des emplois manufacturiers au Japon et $55,7 \%$, au Canada, alors qu'elles représentent $83,5 \%$ de l'ensemble des emplois en Italie et $65,6 \%$, en France. 
documentation, à l'identification d'un certain nombre de publications importantes traitant spécifiquement du JAT en contexte de PME, ce qui devrait être d'une certaine utilité puisque jusqu'à maintenant il ne semble pas y avoir eu de publication faisant état d'un tel exercice.

La discussion sur l'opportunité d'implanter le JAT dans les PME s'appuie sur la synthèse de Julien (GREPME, 1994) concernant les caractéristiques et spécificités de la PME. De cette synthèse ressort une typologie des spécificités qui concerne les aspects suivants : la gestion, la spécialisation/ formalisation, le système d'information et la planification stratégique. De plus, à partir des travaux en cours dans le cadre des activités de la Chaire Bombardier en gestion du changement technologique dans les PME (UQTR), il ressort également un cinquième niveau fondamental d'analyse pour le JAT, à savoir la gestion des opérations dans les PME.

\section{La PME et la gestion}

Dans le fonctionnement des PME, le propriétaire-dirigeant (ou l'entrepreneur) est un intervenant aussi important qu'omniprésent (Filion, 1991; Winston et Heiko, 1990 ; Julien et Marchesnay, 1988). De ce fait, et parce que la structure de la PME est souvent plus simple que celle de la GEM, le propriétairedirigeant est susceptible d'être davantage en contact direct avec les éléments opérationnels de son entreprise (approvisionnements, production, contrôle de la qualité , etc.), ce qui se veut un avantage dans l'implantation du JAT pour une PME (Winston et Heiko, 1990).

Mais il faut voir dans le propriétaire-dirigeant plus que sa place dans l'entreprise ; il est avant tout «... celui (ou celle) qui imagine, développe et réalise ses visions » (Filion, 1991). Cela se traduit en objectifs et selon Marchesnay (1986), ce que l'entrepreneur recherche se rapporte à la pérennité et à la survie de son entreprise, à l'indépendance et à l'autonomie ainsi qu'à la croissance et au pouvoir. Pour Gasse (1982), l'entrepreneur a ainsi un grand besoin d'accomplissement et de réussite ; il est créatif et a beaucoup d'initiative, a confiance en lui et fait preuve d'audace, a un fort besoin d'indépendance et d'autonomie, est motivé, énergique et engagé et il aime les risques modérés. D'un autre côté, il est peu porté à déléguer et à consulter, et ses principales fonctions consistent à rechercher l'efficacité et non l'efficience, à trouver et à exploiter le potentiel de l'entreprise, à dénicher des possibilités au-delà des problèmes, à s'adapter à son environnement en prévoyant les changements à venir et à prioriser l'action.

Ces objectifs, de même que ces attitudes et tendances comportementales de l'entrepreneur, peuvent avoir un impact sur l'implantation du JAT et sur le 
cadre fonctionnel régissant les interactions entre les parties concernées. À propos de l'implantation du JAT, bon nombre d'études soulignent l'importance de facteurs tels que l'implantation graduelle (Manoocheri, 1988; Vora et al., 1990 ; Mehra et Inman, 1992), la gestion participative (Safayeni et al., 1991 ; Hiltrop, 1992), le recours à une expertise externe (Mehra et Inman, 1992) et même l'utilisation d'un système d'aide à la décision (Malley et Ray, 1988 ; Mefford, 1991). Or, de façon générale, l'implantation du JAT en tant qu'élément de différenciation dans la recherche d'un avantage concurrentiel peut profiter des objectifs de pérennité et de croissance d'entrepreneurs cherchant à s'adapter à l'environnement en vue des changements à venir. Mais d'un autre côté, la recherche d'indépendance et d'autonomie jumelée à une faible propension à déléguer et à consulter peuvent s'avérer des freins à la mise en place de facteurs de succès propres au JAT comme la gestion participative, le recours à une expertise externe ou l'utilisation d'un système d'aide à la décision. De plus, si l'entrepreneur est plus concerné par la recherche de l'efficacité que de l'efficience, il existe une possibilité pour que l'implantation du JAT soit davantage guidée par la concrétisation rapide des bénéfices attendus, plutôt que par une approche systémique basée sur les facteurs de succès du JAT et visant l'éradication de l'inefficience à tous les niveaux ${ }^{5}$.

En ce qui concerne le cadre fonctionnel régissant les interactions entre les entreprises en contexte JAT, le partenariat PME/GEM est une stratégie relationnelle qui est très fréquemment mentionnée en tant que facteur de succès du JAT (Gélinas et al., 1994b). Le partenariat consiste à instaurer des relations favorisant une prestation supérieure de la part de la PME fournisseur en contrepartie de quoi la GEM affirme concrètement sa fidélité et son support à la PME. Force est d'admettre toutefois que même une stratégie de partenariat ne peut éluder complètement le rapport de force de facto en place dans une relation PME / GEM. Si ce rapport de force crée un déséquilibre trop défavorable pour la PME, le partenariat pourra accentuer la crainte, pour la PME, d'une dépendance unilatérale se traduisant par une diminution de son autonomie et de son pouvoir (St-John et Heriot, 1993 ; Landry et Trudel, 1993). Nous pouvons dès lors envisager que l'attitude du propriétaire-dirigeant envers le JAT pourrait être défensive, car le maillage fournisseur/client pourrait représenter des risques de perte de liberté, d'imposition de normes et de lois ainsi que des risques liés à la divulgation d'informations confidentielles.

5. De nombreux auteurs définissent d'ailleurs le JAT comme étant l'éradication de l'inefficience à tous les niveaux (Manoocheri, 1988; Wilkinson et Oliver, 1989; Willis et Huston, 1990; Golhar et Stamm, 1991 ; Landry et Trudel, 1993). 


\section{La PME et la spécialisation/formalisation}

Les effets du gigantisme ont aujourd'hui révélé les limites de la GEM : peu de flexibilité, irréversibilité des capacités de production et bureaucratie abondante entre autres (GREPME, 1994). Ces effets, liés à la segmentation pointue des marchés (qui favorise la production en petits lots) et aux changements accélérés rendus nécessaires par les mutations rapides des besoins des marchés avals, expliquent notamment l'importance et le renouveau des PME dans l'économie des pays industrialisés. Pour le JAT, la flexibilité des PME est un avantage certain, puisqu'il s'agit, sur le plan opérationnel, d'un des facteurs de succès d'une grande importance (production de petits lots, faibles coûts et courts délais de mise en course, polyvalence des équipements et de la maind'œuvre). Il est en effet reconnu depuis un certain temps déjà que la PME est plus flexible que la GEM et qu'elle a une plus grande capacité de s'adapter face aux changements et aux perturbations de l'environnement (d'Amboise, 1989 ; Raymond et al., 1991).

La flexibilité de la PME peut aussi avoir un impact favorable indirect au regard du JAT par l'atténuation possible des rapports de force avantageant la GEM dans ses relations avec la PME. En effet, si la GEM ne peut facilement acquérir, à l'interne, autant de flexibilité et de capacité de réaction qu'elle le souhaiterait, elle peut toutefois le faire - et est de plus en plus portée à le faire - en se dotant d'un réseau de partenaires (les PME fournisseurs et les sous-traitants) qui va générer de l'externe ce qui ne peut émaner de l'interne. Ce réseautage crée une complémentarité synergique où la performance de chaque membre du réseau devient tributaire, jusqu'à un certain point, de celle des autres membres, ce qui est d'ailleurs un élément de succès dans l'implantation du JAT (Howard, 1991).

Un autre effet de la différence de taille entre PME et GEM se manifeste dans la prise de décision. Sur le plan organisationnel, les PME sont caractérisées par de petites unités de gestion plus autonomes, peu matures et dépendantes des expériences et de l'expertise du propriétaire-dirigeant (Winston et Heiko, 1990). Elles ont un mode de fonctionnement basé sur une structure plus organique que hiérarchique ou mécaniste (Mintzberg, 1989) et, de ce fait, le processus de prise de décision est souvent peu complexe, axé sur l'action immédiate, et comporte un degré de formalisation moindre (d'Amboise, 1989 ; GREPME, 1994). Ces caractéristiques du processus de décision peuvent, à certains égards, s'avérer avantageuses dans l'implantation du JAT, notamment parce qu'elles incitent naturellement à la minimisation de toutes formes de délais dans la démarche d'implantation et à la recherche de solutions dans le but de régler les problèmes à la source. De plus, des études ont démontré que la structure organisationnelle particulière des PME constitue un 
environnement propice à l'interactivité interne et externe (Julien et Marchesnay, 1988). Cette propension à l'interactivité pourrait faciliter l'implantation du JAT dans la mesure où, justement, le JAT exige un degré élevé d'interactivité interne (implication de la main-d'œuvre et gestion participative) et externe (relations étroites avec les marchés en amont et en aval).

\section{La PME et le système d'information}

La taille d'une entreprise peut également avoir des effets sur le plan informationnel. À cet égard, il convient de mettre en évidence la valeur des réseaux développés par le propriétaire-dirigeant d'une PME : ils permettent d'obtenir à moindre coût une large part d'informations facilitant la consolidation de l'entreprise (Fourcade, 1991) et s'inscrivent dans un cheminement susceptible d'orienter le développement de l'entreprise au sein de son environnement immédiat (Pecqueur, 1989). Toutefois, le JAT requiert davantage que de l'information générique concernant l'évolution de l'environnement socio-économique de la PME. Plusieurs facteurs de succès du JAT liés à l'information ont été identifiés : l'échange de données informatisé (Harisson et Voss, 1990; Anvari, 1992); l'automatisation, l'informatisation et la précision de l'information concernant la production (Golhar et Stamm, 1991); la planification des besoinsmatières (MRP), l'informatisation de la gestion des stocks et la précision de l'information concernant les stocks (Billesbach, 1991; Brown et Mitchell, 1991); la précision de l'information concernant la demande (Anvari, 1992) et la qualité de l'information en général. Or les technologies sous-jacentes à la gestion de ces informations sont certes utilisées convenablement par les GEM mais plusieurs études démontrent que les résultats observés dans les GEM ne peuvent être extrapolés aux PME (Alpar, 1989; Raymond et Blili, 1992). En effet, les PME se préoccupent peu de la gestion des technologies de l'information (manque d'expérience ou de connaissances) et sous-utilisent leurs systèmes d'information. Le lien entre l'utilisation des technologies de l'information et la taille des entreprises, qui s'explique principalement par les limites financières des entreprises de moins grandes dimensions (Inman et Mehra, 1990 ; Golhar et al., 1990) ${ }^{6}$, met donc en évidence les lacunes des PME en matière de gestion de l'information face à certains facteurs de succès importants du JAT. Mais ces limites financières, qui pourraient affecter la qualité du

6. Les limites financières, qui se traduisent souvent par une faible liquidité, rendent plus difficiles l'acquisition d'équipements, l'embauche d'experts et la gestion du système informatique. Les ressources financières destinées à la gestion de l'information dépendent, de plus, largement des priorités des propriétaires-dirigeants des PME; l'évolution de l'entreprise sur le plan des technologies de l'information en sera évidemment affectée. 
système d'information, ne font pas en sorte qu'il y ait nécessairement un lien entre ce dernier et la qualité même de l'information. Pour les PME, la proximité des clients et des dirigeants de l'organisation permet, en partie du moins, de suppléer aux carences du système d'information.

\section{La PME et la planification stratégique}

Le cadre stratégique de la PME en est un de réaction et d'adaptation à l'environnement (Raymond et al., 1991) ; la stratégie repose sur la perception des changements à venir pour un horizon de planification qui, généralement, est de deux ans ou moins. La stratégie est le plus souvent intuitive, opportuniste et active, et met en valeur le caractère étapiste de l'entrepreneur ainsi que la flexibilité de la PME. Ce cadre stratégique, et certaines études le confirment (voir Etkin et al., 1990), représente un inconvénient, car il fait en sorte que les PME qui envisagent d'implanter le JAT n'ont souvent qu'une stratégie restreinte de planification dans la démarche requise. Cette planification stratégique restreinte risque de défavoriser la démarche d'évaluation de l'impact des changements occasionnés par le JAT et de conduire à une vision partielle des conséquences de son implantation.

Une autre particularité de la PME en ce qui a trait à sa planification stratégique est qu'elle est de nature incrémentale et qu'elle est tributaire des visions du propriétaire-dirigeant (Winston et Heiko, 1990). Cette particularité, jumelée à un horizon de planification stratégique relativement court (deux ans ou moins) risque d'avoir, d'une part, un effet positif sur l'implantation du JAT, la PME étant susceptible d'adopter naturellement une démarche étapiste et de prendre action dans des délais relativement courts. D'autre part, un effet négatif peut résulter de l'inadéquation entre la portée temporelle de la planification stratégique et la durée réelle d'une démarche complète d'implantation du JAT. Pour des PME qui opèrent selon un mode souvent loin du JAT, il est en effet possible de constater que la totalité du processus d'implantation du JAT se rapporte à un horizon de planification pouvant aller jusqu'à cinq ans ${ }^{7}$. Cette différence entre la portée temporelle de la planification stratégique et l'horizon réel pour l'implantation du JAT peut avoir comme conséquence une estimation peu réaliste des délais à prévoir pour l'implantation et de la période de rentabilisation des investissements reliés au JAT.

7. Dans le cadre des travaux de la Chaire Bombardier en gestion du changement technologique dans les PME, les études de cas et interventions auprès du réseau de fournisseurs de la division Sea-Doo/Ski-Doo de Bombardier (à Valcourt) ont mis en évidence le fait qu'une durée de cinq ans est un horizon de planification réaliste pour des PME qui amorcent leur transition vers le JAT. 
Enfin, mentionnons que les PME subissent un contrechoc particulier de l'adoption du JAT par les GEM. Pour ces dernières, la restructuration de leur réseau de fournisseurs ainsi que les ententes exclusives et à long terme avec ceux-ci (Anvari, 1992 ; Landry et Trudel, 1993 ; St-John et Heriot, 1993) sont deux facteurs de succès du JAT, pour les GEM, qui contribuent à diminuer leur nombre de fournisseurs. Même si de plus en plus les GEM demandent à leurs fournisseurs de passer au mode JAT (Brown et Mitchell, 1991), il reste que de moins en moins de PME se verront octroyer des contrats de la part des GEM. Par exemple, Xerox, dans sa démarche vers le JAT, a réduit le nombre de ses fournisseurs de 5000 à 300 (St-John et Heriot, 1993). Au regard de la planification stratégique, les PME font alors face à une situation les forçant à développer le plus rapidement possible des expertises et compétences distinctives. Selon le modèle de Porter (1986), les GEM exercent une pression sur les PME qui oblige ces dernières à acquérir un avantage concurrentiel par différenciation. Mais il y a plus : les GEM n'acceptent généralement pas que leurs fournisseurs compensent par des coûts supplémentaires cette différenciation ${ }^{8}$. Là où le bât blesse, c'est que les PME doivent, de façon proactive, concrétiser leur planification stratégique par rapport au JAT en engageant des ressources et en consacrant des efforts pour acquérir un avantage concurrentiel par différenciation alors qu'en même temps, elles doivent maintenir leur avantage par les coûts (Porter, 1991) et surmonter leurs difficultés à affecter les ressources nécessaires à la mise en œuvre du JAT (Stamm et Golhar, 1991).

\section{La PME et la gestion des opérations}

La gestion des opérations (GOP) constitue un volet de caractérisation de la PME qui s'avère très important au regard du JAT. Une PME qui considère adopter le JAT doit évidemment concrétiser sa décision d'adopter ce mode de gestion ; il faut l'implanter, le rendre opérationnel. Concernant donc les caractéristiques de la PME face à la GOP, les recherches indiquent, premièrement, que la structure organisationnelle de la PME est très près des opérations (Gupta, 1988). Comme mentionné à la section 3, cette proximité de contact entre l'organisation et les opérations peut se révéler positive dans l'implantation du JAT. Mais cette même étude indique également que le transfert d'informations entre les niveaux organisationnel et opérationnel est moins formalisé pour les PME et que le découpage des diverses responsabilités y est moins précis à cause, d'une part, du nombre restreint de gestionnaires et, d'autre part, du fait que le rôle de gestionnaire de la production est parfois tenu par le propriétaire-dirigeant lui-même.

8. Le contrôle des coûts est un facteur de succès important pour les GEM voulant opérer en JAT (Hong et Hayya, 1992). 
Or, concernant le transfert d'informations entre les niveaux organisationnel et opérationnel, les recherches font état de l'importance, en JAT, de la précision des informations concernant, entre autres, la production et la gestion des stocks (Wilkinson et Oliver, 1989 ; Joshi et Campbell, 1991). Par conséquent, des modes de gestion et de transfert de ces informations qui s'appuient sur des chaînes de communication moins formelles risquent de rendre encore plus difficile la gestion des opérations en $\mathrm{JAT}^{9}$. Pour ce qui est du découpage moins précis des responsabilités en contexte PME, il est possible que cette situation comporte et des avantages et certains inconvénients. Le fait que le nombre restreint de gestionnaires augmente l'implication du propriétairedirigeant face à la GOP est, semble-t-il, tout à fait compatible avec les facteurs de succès du JAT que sont l'engagement envers ce mode de gestion et la participation directe à son implantation. Mais le propriétaire-dirigeant d'une PME qui s'occupe également de gérer les opérations risque, consciemment ou inconsciemment, d'élaborer des stratégies opérationnelles (politiques de production, choix des produits et procédés, conception du système opérationnel) qui sont en superposition avec sa vision stratégique globale. Si cette vision repose sur un horizon de planification relativement court et sur un mode réactif plutôt que proactif (voir section 5), il est alors plausible qu'une GOP assumée par le propriétaire-dirigeant en soit également subordonnée.

Une deuxième caractéristique des PME en ce qui concerne la GOP a trait au fait que les lignes, postes ou ateliers de production sont plus petits et ont un niveau d'activités plus bas que les grandes entreprises. À première vue, et probablement à juste titre, il pourrait s'ensuivre un avantage concernant l'implantation du JAT : plus de flexibilité, capacité de production plus facilement gérable, production de séries plus courtes. Mais d'un autre point de vue, certaines études mettent en évidence que ce qui définit la complexité des problèmes de production ne se rapporte pas nécessairement à la taille de ces problèmes (capacité de production), mais plutôt à la nature des problèmes. Or, les problèmes de GOP dans les PME, surtout en ce qui concerne le système de pilotage (planification et contrôle) sont essentiellement de même nature que pour les grandes entreprises, et donc tout aussi complexes (Blackstone et Cox, 1985 ; Hamilton et Schroeder, 1984 ; Chrisman, 1985a, 1985b, 1985c). Si tel est le cas, il n'y a alors pas de raison de croire que pour le JAT, qui redéfinit la nature des problèmes de GOP, la taille du système opérationnel puisse constituer un avantage (ou un désavantage) pour la PME.

9. La gestion des opérations est d'entrée de jeu reconnue par plusieurs comme un ensemble d'activités relativement complexes où abondent les procédures. De plus, le niveau de complexité n'est pas nécessairement plus faible pour les PME que pour les grandes entreprises (Blackstone et Cox, 1985). 
Enfin, soulignons qu'en ce qui concerne le JAT, bon nombre d'études mentionnent l'importance de l'informatisation et de l'automatisation de la GOP : échange de données informatisé pour les approvisionnements (Anvari, 1992), automatisation et informatisation de la production (McDaniel et al., 1992), système informatisé de gestion des stocks (Brown et Mitchell, 1991) et automatisation du contrôle de la qualité (Golhar et Stamm, 1991). En fait, la nécessité de recourir aux technologies informatisées a certes été, par le passé, un facteur désavantageux pour la PME. Mais aujourd'hui, la baisse significative des coûts des équipements informatiques, la puissance de plus en plus grande d'équipements de plus en plus petits ainsi que des applications de plus en plus flexibles font en sorte que les PME rattrapent leur retard en matière d'informatisation. Des statistiques relativement récentes indiquent, à cet égard, que pour le Québec, $90 \%$ des PME manufacturières utilisent au moins un microordinateur (Grisé, 1989) et que $50 \%$ de ces entreprises possèdent au moins une technologie informatique de production (Julien, 1992). Concernant donc l'informatisation en tant que facteur de succès du JAT, il semblerait que la capacité d'y recourir ne soit plus une caractéristique défavorable des PME.

\section{Conclusion}

Le JAT est un mode de gestion qui a un impact non seulement sur les opérations des entreprises, mais aussi sur les relations entre celles-ci, notamment en ce qui a trait aux PME dans leurs rapports avec les GEM. Ce rapport fait en sorte que, d'une part, les PME fournisseurs et sous-contractants des GEM sont de plus en plus pressées d'adopter le JAT, les GEM désirant, en première instance, y recourir afin d'améliorer leur efficacité, donc leur position concurrentielle. Mais, d'autre part, les recherches et expériences portant sur le JAT démontrent que ce mode de gestion conduit éventuellement à une réduction du nombre de fournisseurs avec qui transigent les GEM (St-John et Heriot, 1993). Il y a donc beaucoup d'appelés, mais peu d'élus. Devant une telle situation, les PME qui désirent conserver leurs clients, souvent importants, que sont les GEM, n'ont d'autre choix que de s'adapter. Cette adaptation peut-elle alors être favorablement ou défavorablement affectée par les caractéristiques et spécificités de la PME ? C'est à cette question que nous avons tenté de répondre. Le tableau 1 résume les éléments de discussion abordés dans les sections précédentes et indique si les caractéristiques et spécificités considérées sont favorables ou défavorables au JAT.

Ce qui ressort principalement de cette analyse est que ce qui fait justement la force de la PME peut parfois constituer des faiblesses dans un processus d'implantation du JAT. Ainsi, la recherche de l'efficacité plutôt que de l'efficience peut être positive en ce qui concerne une action rapide et la 
TABLEAU 1

Caractéristiques et spécificités des PME : adéquation avec le JAT

Particularités et spécificités de la PME

L'importance et l'omniprésence du propriétaire-dirigeant induit un contact direct avec les opérations.

Objectifs de pérennité et de croissance de l'entrepreneur.

Recherche d'indépendance jumelée à une faible propension à déléguer et à consulter.

Recherche de l'efficacité plutôt que de l'efficience.

Recherche de l'autonomie et de l'indépendance.

Flexibilité de la PME.

Simplicité du processus de prise de décision axé sur l'action immédiate.

La structure organisationnelle de la PME qui est propice à l'interactivité interne et externe.

Sous-utilisation des technologies de l'information.
Favorable ou défavorable

Avantage pour l'implantation du JAT.

Favorable en général.

Freins pour la mise en place de facteurs de succès spécifiques au JAT comme la gestion participative, le recours à une expertise externe ou l'utilisation d'un système d'aide à la décision.

Favorise la recherche de bénéfices à court terme pour le JAT plutôt qu'une approche systémique basée sur les facteurs de succès du JAT et visant à éliminer toute forme d'inefficience.

Défavorable si le propriétaire-dirigeant perçoit le renforcement des relations PME/GEM en JAT comme représentant des risques de perte de liberté, d'imposition de normes et de divulgation. d'informations confidentielles.

Avantage sur le plan opérationnel, car il s'agit d'un facteur de succès important pour le JAT.

Favorable aussi, car la flexibilité a un impact indirect sur le rapport de force GEM/PME, en faveur de la PME; celle-ci procure de l'externe la flexibilité que la GEM ne peut se procurer de l'interne.

Favorable à l'implantation du JAT parce que cette caractéristique incite naturellement à la minimisation des délais et à la recherche de solutions dans le but de régler les problèmes à la source.

Facilite l'implantation du JAT qui, justement, exige un degré élevé d'interaction interne (implication de la main-d'oeuvre et gestion participative) et externe (relations étroites avec les marchés amont et aval).

Défavorable pour le JAT au regard de facteurs de succès tels que l'échange de données informatisé, la production et la planification informatisées et la qualité de l'information en général. 
TABleAu 1 (suite)

Caractéristiques et spécificités des PME : adéquation avec le JAT

\section{Particularités et spécificités} de la PME

Planification stratégique qui porte sur un horizon relativement court.

Planification stratégique incrémentale et à court terme.

Transfert d'information moins formalisé entre les niveaux organisationnels et opérationnels.

Découpage moins précis des responsabilités à cause, d'une part, du nombre restreint de gestionnaires et, d'autre part, du fait que le rôle de gestionnaire de la production est parfois assumé par le propriétairedirigeant.

Petite taille des lignes, postes ou ateliers de production.

\section{Favorable ou défavorable}

Défavorable en ce qui concerne l'évaluation de l'impact des changements occasionnés par le JAT.

Favorable pour l'implantation du JAT, car la PME est susceptible d'adopter naturellement une démarche étapiste et de prendre action dans des délais relativement courts.

Défavorable, car il peut y avoir inadéquation entre la portée temporelle de la planification stratégique et la durée réelle d'une démarche complète d'implantation du JAT.

Risque de rendre plus difficile la GOP en mode JAT.

Le nombre restreint de gestionnaires peut être un avantage pour le JAT en favorisant l'implication des gestionnaires principaux (propriétairedirigeant) face à la GOP.

Peut aussi être un désavantage à cause de la superposition de la vision stratégique globale du propriétaire-dirigeant avec les stratégies opérationnelles.

Peut être un avantage pour le JAT des points de vue de la flexibilité, de la gestion des capacités de production, de séries de production plus courtes, etc.

Peut ne pas constituer un avantage (ou un désavantage) dans la mesure où le JAT redéfinit la nature des problèmes et non leur taille.

Semble en voie de ne plus être un désavantage pour les PME.

Accès à l'informatisation.

résolution de problèmes à la source, mais il peut aussi y avoir un risque de ne rechercher que des bénéfices à court terme au détriment d'une approche plus systémique axée sur la réussite à long terme.

Certaines caractéristiques de la PME témoignent de son dynamisme et de son opportunisme en vue de l'implantation du JAT. En général, il semblerait que la flexibilité des PME, les objectifs de pérennité et de croissance de l'entrepreneur, la simplicité du processus décisionnel ainsi que la proximité 
des niveaux organisationnels et opérationnels de la PME soient des caractéristiques normalement compatibles avec le JAT. D'un autre côté, la recherche de l'efficacité plutôt que de l'efficience, la sous-utilisation des technologies de l'information et une planification stratégique portant sur un horizon à court terme seraient des caractéristiques pouvant être défavorables dans une démarche d'implantation du JAT. Il faut bien comprendre que ces dernières caractéristiques peuvent éventuellement s'avérer défavorables, mais qu'elles ne le seront pas nécessairement si des efforts sont faits afin, d'une part, d'en prendre conscience et, d'autre part, d'adopter une vision plus systémique de l'entreprise et de son évolution vers le JAT.

Mentionnons de plus que d'autres caractéristiques des PME telles que le contact plus direct des dirigeants avec les employés ou une disponibilité en ressources financières moins grande que pour les GEM pourraient être mises de l'avant comme étant respectivement favorable et défavorable au JAT. Ce sont des caractéristiques pour lesquelles l'argumentation est relativement triviale et bien documentée. Mais d'autres éléments de discussion pourraient faire l'objet de recherches futures parce que, justement, la documentation actuelle en traite peu ou pas du tout. Entre autres, il semblerait pertinent d'étudier l'impact que pourraient avoir les relations patronales / syndicales sur le processus d'implantation du JAT en PME. Enfin, plusieurs PME font face à une forte dépendance à un client unique (souvent une GEM). Cette dépendance, qui se concrétise par un pourcentage important des revenus de la PME fourni par un seul client pourrait, bien que cette hypothèse demeure à vérifier, constituer une caractéristique défavorable par rapport au JAT en ce qu'elle dicterait une démarche d'implantation plus ou moins forcée et à un rythme plus ou moins compatible avec les possibilités de la PME concernée.

\section{Bibliographie}

ACS, Z.J. (1992), «Small business economics : a global perspective», Challenge, novembre-décembre, p. 38-44.

ALPAR, P. (1989), "The role of information systems in entrepreneurial firms ", Document $\mathrm{n}^{0} 89-3$, Centre de recherche sur la gestion de l'information, Chicago, Université d'Illinois à Chicago.

ANVARI, M. (1992), «Electronic data interchange and inventories », International Journal of Production Economics, vol. 26, p. 135-143.

BÉRARD, D. (1992), «Les esclaves du Juste-à-Temps », Commerce, janvier, p. 61-65.

BILlESBACH, T.J. (1991), «A study of the implementation of JiT in the United-States », Production and Inventory Journal, $3^{\mathrm{e}}$ trimestre, p. 1-4. 
BLACKSTONE, J.H. et J.F. CoX (1985), « Are small manufacturers ready for MRP?», Comptes rendus de la $27^{e}$ conférence internationale annuelle de l'APICS, p. 3-6.

Brown, K.A. et T.R. Mitchell (1991), «A comparison of Just-in-Time and batch manufacturing : the role of performance obstacles », Academy of Management Journal, vol. 34, nº 4, p. 906-917.

ChaIRE Bombardier SEA-Doo/ SKI-DOo EN GESTION DES CHANGEMENTS TECHNOLOGIQUES DANS LES PME, (1995), «Le Juste-à-Temps : de la philosophie à la pratique », Rapport de session préparé par le Groupe de recherche en économie et gestion des PME, Université du Québec à Trois-Rivières.

Chrisman, J.J. (1985a), «Basic production techniques for small manufacturers: 1. Initial preparations », Production and Inventory Management, $2^{\mathrm{e}}$ trimestre, p. $130-145$.

Chrisman, J.J. (1985b), «Basic production techniques for small manufacturers : 2. Inventory control methods and $\mathrm{MRP} »$, Production and Inventory Management, $3^{\mathrm{e}}$ trimestre, p. 48-63.

ChRISMAN, J.J. (1985c), «Basic production techniques for small manufacturers: 3. Production planning, control and scheduling methods », Production and Inventory Management, $4^{\mathrm{e}}$ trimestre, p. 14-26.

D’Amboise, G. (1989), La PME canadienne : situation et défis, Québec, Les Presses de l'Université Laval.

DElmas, P. et G. RoY (1988), «Industries américaines : la faiblesse au cœur », Économie Prospective Internationale, $4^{\mathrm{e}}$ trimestre, $\mathrm{n}^{\mathrm{0}} 36$, p. 91-116.

ETKIN, L.P., F. RAISZADEH et H.R. HUNT (1990), «Just-in-Time : a timely opportunity for small manufacturers », Industrial Management, janvier-février, p. 16-18.

FILION, L.J. (1991), Vision et relations : clefs du succès de l'entrepreneur, Montréal, Les Éditions de l'Entreprise.

FourCADE, C. (1991), Petite entreprise et développement local, Paris, Eska.

GASSE, Y. (1982), «L'entrepreneur moderne : attributs et fonctions », Gestion, vol. 7, $\mathrm{n}^{\circ} 4$, p. 3-10.

GÉlinas, R., R. JACOB, J. DROLET et M. RheAult (1994a), «Les facteurs de succès du Juste-à-Temps et leur incidence sur les PME », Comptes-rendus de l'ICSB, juin, p. 141-151.

GÉlinas, R., R. JaCob, J. Drolet et M. Rheault (1994b), «Une approche systémique pour l'évaluation des PME en vue de l'implantation du Juste-à-Temps », Comptes-rendus de l'ASAC, vol. 15, $\mathrm{n}^{0} 7$, p. 37-46.

GolhaR, D.Y. et C.L. STAMm (1991), «The Just-in-Time philosophy : a literature review », International Journal of Production Research, vol. 29, n 4, p. 657-676. 
GolhaR, D.Y. et C.L. STAMM et W.L. SMITH (1990), « JiT implementation in small manufacturing firms», Production and Inventory Management Journal, $2^{\mathrm{e}}$ trimestre, p. 44-48.

GREPME (1994), Les PME: bilan et perspective, Québec, Les Presses Inter Universitaires.

GRISÉ, J. (1989), L'informatisation du Québec : profil de la demande, Québec, Les Publications du Québec.

GUPTA, Y.P. (1988), «Linking small business and modern management techniques », Industrial Management and Data Systems, mars-avril, p. 13-19.

Hamilton, S. et R. Schroeder (1984), «Computer-based manufacturing and accounting systems for smaller manufacturing firms », Production and Inventory Management, vol. 25, $\mathrm{n}^{0} 4$, p. 92-105.

HARISSON, A. et C. Voss (1990), «Issues in setting up JiT supply », International Journal of Operations and Production Management, vol. 10, $\mathrm{n}^{\circ} 2$, p. 84-93.

HILTROP, J.M. (1992), «Just-in-Time manufacturing: implications for the management of human resources », European Management Journal, vol. 10, n ${ }^{0} 1$, p. 49-55.

HONG, J.D. et J.C. HAYYA (1992), « Just-in-Time purchasing : single or multiple sourcing ?», International Journal of Production Economics, vol. 27, p. 175-181.

HowARD, R. (1991), «Can small business help countries compete», Harvard Business Review, novembre-décembre, p. 88-103.

INMAN, R.A. et S. MEHRA (1990), «The transferability of Just-in-Time concepts to American small business », Interfaces, vol. $20, \mathrm{n}^{0} 2$, p. 30-37.

JoSHI, K. et J.F. CAMPBELL (1991), « Managing inventories in a JiT environment », International Journal of Purchasing and Materials Management, avril, p. 32-36.

JULIEN, P.A. (1992), «Les nouvelles technologies dans les PME manufacturières québécoises », Gestion, vol. 17, nº 4, p. 29-38.

JULIEN, P.A. et M. MARCHESNAY (1988), La petite entreprise : principes d'économie et de gestion, Paris, Vuibert, Collection Gestion.

LANDRY, P. (1990), «Just-in-Time : managing your suppliers », Purchasing World, janvier, p. 44-47.

LANDRY, S. et Y. TRUDEL (1993), «Les approvisionnements en juste-à-temps : les risques d'abus », Gestion, février, p. 6-11.

Light, L. (1993), «Small businesses : the job engine needs fuel », Business Week, $1^{\mathrm{er}}$ mars, p. 78.

MANOOCHERI, G.H. (1988), «Just-in-Time for smaller manufacturer », Journal of Small Business Management, octobre, p. 22-30. 
MALLEY, J.C. et R. RAY (1988), «Informational and organizational impacts of implementing a JiT system », Production and Inventory Management, $2^{\mathrm{e}}$ trimestre, p. 66-70.

MARCHESNAY, M. (1988), «La mercatique de la petite entreprise», Revue Internationale $P M E$, vol. $1, \mathrm{n}^{\text {os } 3-4, ~ p . ~ 259-276 . ~}$

Marchesnay, M. (1986), La stratégie, Paris, Chotard.

MCDaniel, S., J.G. Hormsby et A.B. Gresham (1992), « The effect of JiT on distributors », Industrial Marketing Management, vol. 21, p. 145-149.

MEFFORD, R.N. (1991), «Quality and productivity : the linkage », International Journal of Production Economics, vol. 24, p. 137-145.

Mehra, S. et R.A. InMAN (1992), «Determining the critical elements of Just-in-Time implementation », Decision Sciences, vol. 23, n 1 , p. 160-174.

Mintzberg, H. (1989), Mintzberg on Management: Inside Our Strange World of Organizations, New York, The Free Press.

OfFodile, O.F. et D. ARRINGTON (1992), «Support of successful Just-in-Time implementation : the changing role of purchasing », International Journal of Physical Distribution \& Logistics Management, vol. 22, $\mathrm{n}^{0} 5$, p. 38-46.

PECQUeUR, B. (1989), «Un réseau ne se crée pas par défaut », dans M. Gault (éd.), Ville intermédiaire pour l'Europe, Paris, Syros / Alternatives.

PORTER, M. (1991), « Towards a dynamic theory of strategy », Strategic Management Journal, vol. 12, p. 95-117.

PORTER, M. (1986), Choix stratégique et concurrence, Paris, Economica.

PotTer, P. (1990), «JiT turns suppliers into partners », Systems/3X \& AS World, vol. 10 , p. $128-132$.

RAYMOND, L., F. BERgeron, L. GingraS et S. Rivard (1991), «Problématique de l'informatisation dans les PME», Technologie de l'information et Société, vol. $3, \mathrm{n}^{\circ} 1$, p. 131-148.

RAYMOND, L. et S. BLILI (1992), «Les systèmes d'information dans les PME: synthèse et apports de la recherche », Revue Organisation, été, p. 146-166.

Safayeni, F., L. Purdy, R. VAn Engelen et S. Pal (1991), «Difficulties of Justin-Time implementation: a classification scheme», International Journal of Operations and Production Management, vol. 11, p. 27-36.

STAMM, C.L. et D.Y. GolHAR (1991), « Customer and supplier linkages for small JiT manufacturing firms », Journal of Small Business Management, juillet, p. 43-49.

ST-John, C.H. et K.C. HeRIOT (1993), «Small suppliers and JiT purchasing», International Journal of Purchasing and Materials Management, hiver, p. 11-16. 
VorA, J.A., J.V. SARAPH et D.L. PETERSON (1990), « JiT implementation practices », Production and Inventory Management Journal, $3^{\mathrm{e}}$ trimestre, p. 57-59.

Wilkinson, B. et N. Oliver (1989), « Power, control, and the Kanban », Journal of Management Studies, vol. 26, $\mathrm{n}^{0} 1$, p. 47-58.

WILlis, T.H. et C.R. HUSTON (1990), « Vendor requirements and evaluation in a JiT environment », International Journal of Operations and Production Management, vol. $10, \mathrm{n}^{\mathrm{0}} 4$, p. $41-50$.

WinStON, R. Jr. et L. HEIKo (1990), «Just-in-Time and small business evolution », Entrepreneurship: Theory and Practice, été, p. 51-64. 